\title{
Prevalencia de Lesiones Ateroscleróticas Carotídeas en Radiografía Panorámica y su Relación con Factores de Riesgo Cardiovascular en Brasileños
}

\author{
Prevalence of Atherosclerotic Carotid Lesions on Panoramic Radiography \\ and Relationship with Cardiovascular Risk Factors in Brazilians
}

Garbelotti Junior, S. ${ }^{\text {; }}$ Ribeiro Moreira, W. ${ }^{2}$; Aguiar, H. ${ }^{2}$; Olave, E. ${ }^{3}$ \& Queiroz, D. ${ }^{2}$

GARBELOTTI JUNIOR, S.; RIBEIRO MOREIRA, W.; AGUIAR, H.; OLAVE, E. \& QUEIROZ, D. Prevalencia de lesiones ateroscleróticas carotídeas en radiografía panorámica y su relación con factores de riesgo cardiovascular en brasileños. Int. J. Morphol., 38(6):1560-1565, 2020.

RESUMEN: Aunque la radiografía panorámica no es una prueba de diagnóstico inicial para detectar la aterosclerosis carotídea, no podemos ignorar su valor al ver estas lesiones como hallazgos incidentales que pueden ser grandes aliados en el diagnóstico temprano y la prevención de eventos vasculares graves. El objetivo de este estudio fue evaluar la prevalencia de calcificaciones en la bifurcación de la arteria carótida en las radiografías panorámicas de rutina como principio diagnóstico, así como su relación con los factores de riesgo cardiovascular en individuos brasileños. Se analizaron 449 exámenes de radiografía panorámica de la base de datos del Departamento de Radiología de la Facultad de Odontología de Piracicaba, UNICAMP, São Paulo, Brasil. Después de obtener mediciones de la presión arterial e información sobre el tabaquismo y las enfermedades sistémicas, fue buscado en la radiografía panorámica la presencia o ausencia de la masa radiopaca en las regiones de las vértebras cervicales C3-C4. En el caso de una imagen sugestiva de ateroma, se realizó una radiografía anteroposterior para confirmar el diagnóstico. Aplicamos la prueba estadística de Chi-cuadrado con distribución normal y muestras independientes y aleatorias con un intervalo de confianza del $95 \%$. La confirmación de calcificaciones carotideas se produjo en el 8,5\% de la población de estudio (38 casos). No encontramos ninguna relación entre la presencia de calcificaciones con el tabaquismo, con el sexo o con datos previos de antecedentes familiares. Sin embargo $49,1 \%$ de los casos estaban por encima de sesenta años y tenía asociación significativa con la hipertensión $(\mathrm{P}=0,006)$. El mayor riesgo se presenta en pacientes ancianos hipertensos y, por lo tanto, hacemos hincapié en la importancia de una observación minuciosa de las radiografías panorámicas en la búsqueda de cualquier alteración de la anatomía normal y si es así, proceder con la derivación lo antes posible para un diagnóstico concluyente por parte de un especialista, pudiendo así ayudar en la prevención de episodios vasculares graves.

PALABRAS CLAVE: Aterosclerosis; Arteria carótida; Radiografía panorámica; Hipertensión.

\section{INTRODUCCIÓN}

Cada año, 17 millones de personas sufren un derrame cerebral en todo el mundo, 6,5 millones mueren y 26 millones viven con discapacidad permanente (Conselho Nacional de Secretários de Saúde, 2019). Los accidentes cerebrovasculares representan un grave problema de salud pública, ya que causan secuelas significativas o incluso la muerte, se estima que para 2050, tanto en el primer mundo como en América Latina, el número de personas afectadas aumentará hasta un $59 \%$. Esta es la tercera causa de muerte en todo el mundo y la principal causa de discapacidad, y aproximadamente el $60 \%$ de los supervivientes tienen de- ficiencias incapacitantes generando altos costos para el tratamiento y la rehabilitación de estos pacientes (Almog et al., 2004; Roldán-Chicano et al., 2006; Ramesh \& Pabla, 2007; Bengtsson et al., 2014; Atalay et al., 2015; Chacón, 2016).

Estadísticas del gobierno brasileño indican que el accidente cerebrovascular es la principal causa de muerte y discapacidad en la población adulta, se registran 68,000 muertes por accidente cerebrovascular anualmente $(10 \%$ del total de muertes) y es responsable del $10 \%$ de las

\footnotetext{
${ }^{1}$ Departamento de Morfologia e Genética, Pós-graduação em Biologia Funcional e Estrutural - Universidade Federal de São Paulo, São Paulo, Brasil.

${ }^{2}$ Departamento de Radiologia da Faculdade de Odontologia de Piracicaba - Universidade Estadual de Campinas, São Paulo, Brasil.

${ }^{3}$ Facultad de Medicina, Universidad de La Frontera, Temuco, Chile.
} 
admisiones en los hospitales públicos. Además, Brasil tiene la cuarta tasa de mortalidad por accidente cerebrovascular entre los países de América Latina y el Caribe (Bensenor $e t$ al., 2015; Ministério da Saúde do Brasil, 2019).

Se sabe que aproximadamente el $20 \%$ de los accidentes cerebrovasculares se deben a una embolia cardiogénica, sin embargo, aproximadamente el $30 \%$ siguen siendo idiopáticos después de una extensa investigación etiológica y la circulación más comúnmente afectada es la arteria carótida, que corresponde al $80 \%$ de los casos (Abreu et al., 2015).

La aterosclerosis es una enfermedad inflamatoria crónica con una naturaleza multifactorial, caracterizado por el engrosamiento y pérdida de elasticidad de las paredes arteriales, y es responsable de la mayoría de los accidentes cerebrovasculares, ya sea isquémico o hemorrágico (Friedlander et al., 2005; Bengtsson et al.). La formación de placas ateroscleróticas comienza con lesiones endoteliales causadas por factores como el estrés y ciertos hábitos como la inactividad física, el tabaquismo, la dieta, la obesidad, la hipertensión y la diabetes como factores relevantes para el establecimiento de la enfermedad, además del perfil socioeconómico y la edad avanzada (Bengtsson et al.; Yoon et al., 2014; Abreu et al.; Atalay et al.; Johansson et al., 2015; Chacón et al.).

Calcificación arterial en grandes vasos fuera del cerebro se ha asociado con diversas enfermedades cerebrales (Chacón et al.). Gran parte de las calcificaciones de los tejidos blandos en la región de la cabeza y el cuello pueden eventualmente detectarse mediante radiografía panorámica, un examen ampliamente utilizado por los dentistas y que requiere un amplio conocimiento anatómico de las estructuras craneofaciales y cervicales de los profesionales para diferenciar los posibles hallazgos incidentales correctamente, tales como el ateroma calcificado en las arterias carótidas, que requieren investigación médica especializada y urgente porque representan un riesgo para la vida del paciente, debido a la relación directa con el accidente cerebrovascular (Almog et al., 2004; Roldán-Chicano et al.; Ramesh \& Pabla; Borba et al., 2016).

Aunque muchos estudios han informado calcificaciones carotídeas en muchas poblaciones, solo unos pocos como Ngamsom et al. (2015), se centró en la relación entre los casos positivos y la historia clínica del paciente. Por lo tanto, el objetivo del presente estudio fue evaluar la prevalencia de calcificaciones en la bifurcación de la arteria carótida en radiografías panorámicas de rutina, así como su correlación con factores de riesgo cardiovascular en un grupo de brasileños.

\section{MATERIAL Y MÉTODO}

Este es un estudio retrospectivo para el cual se seleccionaron y evaluaron los registros digitales de 449 pacientes mayores de treinta años que asistieron espontáneamente a una radiografía panorámica de rutina solicitada por un dentista (Tabla I). Este trabajo fue presentado al Comité de Ética e Investigación, siendo aprobado y archivado por el $\mathrm{n}^{\circ}$ 366.012 de 14.08.2013.

Tabla I - Datos demográficos.

\begin{tabular}{lc}
\hline & $\mathrm{n}=449$ \\
\hline Edad (años) & $50,17(11,13)$ \\
Masa corporal $(\mathrm{kg})$ & $74,52(15,38)$ \\
Estatura $(\mathrm{m})$ & $1,64(0,1)$ \\
Indice de masa corporal $\left(\mathrm{kg} / \mathrm{m}^{2}\right)$ & $27,65(4,94)$ \\
Circunferencia de la cintura $(\mathrm{cm})$ & $95,96(12,95)$ \\
Presión arterial (mm Hg) & $122,5(16,13) / 78,6(11,44)$ \\
Datos promedio (desviación estándar). & \\
\hline
\end{tabular}

Los exámenes fueron parte del tratamiento de los pacientes y siguieron un protocolo de rutina en cuatro etapas: 1) Examen clínico en el que obtuvimos mediciones de la presión arterial y antecedentes médicos individuales y familiares; 2) de radiografía panorámica, usando un aparato de imagen analógica convencional (OP 100® Instrumentarium Corp. Imaging Division, Finland); 3) Evaluación de la imagen panorámica por un dentista especializado en radiología, que identificó la presencia o ausencia de una masa radiopaca irregular en la región de las vértebras cervicales $\mathrm{C} 3$ y $\mathrm{C} 4$; 4) Los pacientes que presentaron una imagen sugestiva de ateroma (Fig. 1), se sometieron a una radiografía complementar para diferenciar el diagnóstico, a través de una radiografía anteroposterior con la cabeza inclinada hacia atrás, en una posición más apropiada del cuello (AP de Towner modificado), con un bajo factor de exposición que facilita la visualización de la calcificación y permite la diferenciación de otras estructuras radiopacas adyacentes (Fig. 2).

En los casos positivos, el dentista hizo un informe y los pacientes fueron remitidos para una evaluación médica específica que podría anticipar posibles eventos cerebrales o cardiovasculares.

Para evaluar cuantitativamente la relación entre la frecuencia de la presencia de calcificaciones y la distribución esperada del fenómeno con respecto a los factores de riesgo cardiovascular. Se realizó la prueba de Chi-cuadrado de distribución normal y muestras independientes y aleatorias y las poblaciones deberían tener una variación común. El cálculo se realizó con la ayuda del software SPSS 20,0 (SPSS Inc., Chicago, IL), y se consideró el intervalo de confianza del $95 \%(\mathrm{p}<0,05)$, que indicó significancia. 


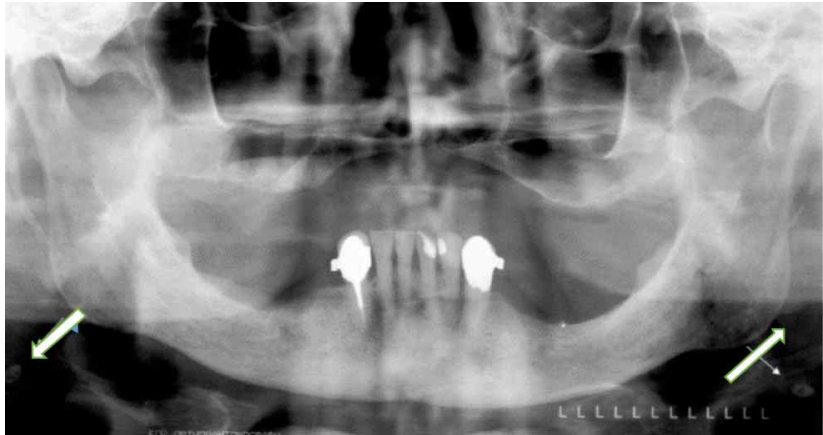

Fig. 1. Radiografía panorámica, imagen de ateromas bilaterales (fuente propia).

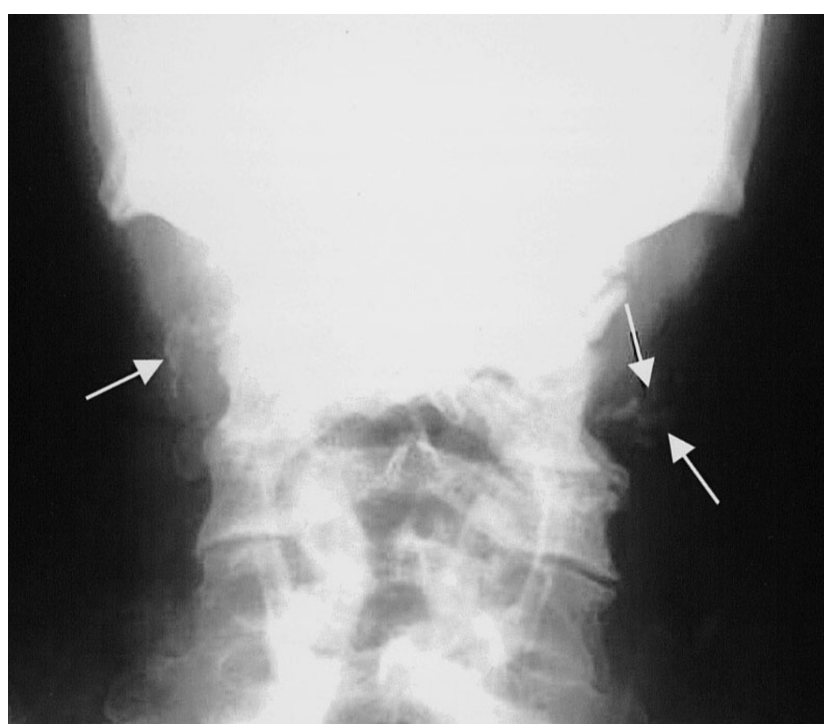

Fig. 2. Radiografía anteroposterior complementaria (AP Towner modificada) con imagen de ateromas bilaterales (fuente propia).

\section{RESULTADOS}

La Tabla I muestra los datos demográficos de los pacientes que participaron en la muestra del estudio.

La Tabla II presenta la distribución muestral de casos de aterosclerosis. La confirmación de calcificaciones carotídeas se produjo en el 8,5\% de la población estudiada (38 casos). No hubo relación entre la presencia de calcificaciones con el tabaquismo, con el sexo o con los datos anteriores de la historia familiar. Sin embargo, observamos datos relevantes, prácticamente la mitad de los casos $(49,1 \%)$ ocurrieron por encima de los sesenta años y hubo una relación significativa con la hipertensión $(\mathrm{P}=0,006)$. También observamos que la aparición de la calcificación mostró predominio unilateral, con $63,15 \%$ de los casos.

\section{DISCUSIÓN}

El factor principal que diferencia al profesional de la salud es la sutileza de obtener diagnósticos precisos. Al llevar a cabo un tratamiento adecuado en el campo dental, el examen radiográfico adicional oral, la radiografía panorámica de la cara ha sido utilizado por el dentista como un método auxiliar para el diagnóstico de enfermedades orales. A pesar de las grandes ventajas mencionadas en este estudio, con respecto a los grandes beneficios de la radiografía panorámica, muchos profesionales aún desconocen que en este examen se puede identificar la aterosclerosis en la bifurcación de la arteria carótida. (Bayer et al., 2011).
Tabla II. Prevalencia y distribución de casos positivos de visualización de la calcificación carotídea en radiografías panorámicas, y relación con factores de riesgo cardiovascular.

\begin{tabular}{lccccc}
\hline \multirow{4}{*}{ Sexo } & & $\mathrm{n}$ & Calcificación & Incidencia & p-valor \\
\hline \multirow{4}{*}{ Predominio } & Hombre & 449 & 38 & 8,46 & \\
& Mujer & 274 & 11 & 6,28 & 0,185 \\
& Unilateral & & 27 & 9,85 & \\
& Bilaterales & & 24 & 63,15 & 0,241 \\
Edad & $30-40$ & 90 & 14 & 36,85 & - \\
& $41-50$ & 120 & 4 & 1,11 & - \\
& $51-60$ & 139 & 13 & 3,34 & - \\
\multirow{4}{*}{ Hipertensión } & $61-70$ & 87 & 16 & 18,35 & - \\
& $>70$ & 13 & 4 & 30,76 & - \\
Tabaquismo & No & 322 & 20 & 6,21 & $0,006^{*}$ \\
& Yes & 127 & 18 & 14,17 & \\
Historia familiar & No & 361 & 27 & 7,47 & 0,129 \\
& Yes & 88 & 11 & 12,5 & \\
* Qui-cuadrado; $\mathrm{P}<0,05$. & No & 325 & 25 & 7,69 & 0,342 \\
\hline
\end{tabular}

Desde que Friedlander \& Lander (1981) publicó una encuesta en 1981, donde utilizaron radiografía panorámica para visualizar calcificaciones en la arteria carótida, se han realizado muchos estudios con la intención de mostrar la importancia del análisis amplio de esta prueba para la detección de calcificaciones en la arteria carótida. De hecho, varios estudios han demostrado que esta técnica convencional tiene un valor relativamente grande, en la visualización de calcificaciones se ha demostrado en estudios que enfatizan la importancia del diagnóstico para la pre- 
vención de posibles eventos cardiovasculares (Ravon et al., 2003; Almog et al., 2005; Almog, 2007; Friedlander et al., 2005; Garoff et al., 2016; Gonçalves et al., 2016).

Pero para ser efectiva la visualización, el profesional debe tener mucha habilidad y un amplio conocimiento de la interpretación diagnóstica en la evaluación de la radiografía de la calcificación de distinguir de otras estructuras anatómicas o patológicas.

Vale la pena señalar que la radiografía panorámica no es prueba de diagnóstico para la detección temprana de la aterosclerosis carotídea, y calcificaciones en los tejidos blandos que aparecen son por lo general los hallazgos incidentales, por lo que es importante que el profesional sepa como evaluar e interpretar estas imágenes y no confundir con otras estructuras (Almog et al., 2005; Atalay et al.; Borba et al.; Abreu et al.).

Aunque Ravon et al., Friedlander et al. (2005) y Lee et al. (2014) afirmaron que las calcificaciones observadas en las radiografías panorámicas se asociaron positivamente con la aterosclerosis carotídea, según estudios de Madden et al. (2007), Yoon et al. (2008) y Bastos et al. (2012) mostraron baja sensibilidad para detectar calcificaciones carotídeas y afirman estar de acuerdo con Almog e Imanimoghaddam et al. (2012) para el estudio vascular extracraneal de la carótida, la ecografía Doppler se considera el "estándar de oro" de las técnicas no invasivas para determinar la calcificación y el grado de estenosis causadas por la enfermedad aterosclerótica y concluyó que, debido a la baja sensibilidad y al valor predictivo positivo, la radiografía panorámica no puede considerarse una prueba precisa o confiable para calcificación o estenosis.

A pesar de esto, el valor de la radiografía panorámica cuando se visualizan calcificaciones carotídeas no debe disminuirse. La radiografía panorámica de la cara es una prueba de bajo costo y, aunque no debe usarse como método de diagnóstico inicial en la búsqueda de calcificaciones carotídeas, puede ser una herramienta importante para detectar calcificaciones en las arterias carótidas (Almog et al., 2004; Roldán-Chicano et al.; Ramesh \& Pabla; Atalay et al.; Borba et al.).

Por lo tanto, los dentistas deben conocer la posibilidad, particularmente en pacientes de edad avanzada o aquellos con factores de riesgo cardiovascular como obesidad, diabetes, hipertensión y tabaquismo, y observar cuidadosamente la región y si es necesario, derivar al paciente incluso si es asintomático para investigación médica, pudiendo reducir así la morbilidad y mortalidad de esta población. (Ramesh \& Pabla; Bayer et al.; Friedlander et al., 2013;
Moshfeghi et al., 2014; Atalay et al.; Johansson et al.; Borba et al.; Chacón et al.).

Los datos del Ministerio de Salud de Brasil han mostrado que el accidente cerebrovascular tiene una mayor incidencia con mayor mortalidad y discapacidad en los brasileños que el promedio mundial, por lo que una hipótesis era que los brasileños tenían una mayor tasa de calcificación carotídea en la misma proporción. Pero lo que vemos es que la incidencia de hallazgos incidentales en la población de estudio con un total de $8,46 \%$ no es muy diferente de otros estudios como Lee et al. (2013, 2014), Atalay et al., Garoff et al. y Gonçalves et al., quienes encontraron entre 6 y $7 \%$ de incidencia, pero casi dos veces según lo informado por Almog et al. $(2004,2005)$ informaron de 3 a $5 \%$, Bayer et al. con 4,8 \%, Friedlander et al. $(2005,2013)$ con 4,2 \% y Cohen et al. (2002) con $3,8 \%$ de casos positivos.

Es un hecho que uno de los factores de riesgo más importante es la edad y nuestros resultados muestran que casi dos tercios de los casos identificados ocurrieron en el grupo de edad de más de 50 años. Datos similares a la mayoría de los estudios como Cohen et al., Moshfeghi et al. y Gonçalves et al., informaron prevalencia en pacientes con una edad promedio de $\sim 50$.

En general, nuestros resultados siguen la tendencia reportada en la literatura, donde a mayor edad, mayor es el riesgo de hallazgos radiopacos compatibles con lesiones ateroscleróticas (Bengtsson et al.), ya que casi la mitad de nuestros casos ocurrieron por encima de sesenta años.

En este sentido, algunos estudios tienen una incidencia similar, pero edades medias mucho más altas, según lo presentado por Friedlander et al. (2013) con 4,2 \% y 73,2 años, Lee et al. (2013) con 6,2 \% y 67,4 años, Lee et al. (2014) con $6 \%$ y 64,9 años, Atalay et al. con $6 \%$ y 59,84 años y Garoff et al. con $7 \%$ e 68 años.

Al igual que Lee et al. (2014) e Gonçalves et al., tampoco encontramos diferencias significativas entre los sexos. En cuanto a los antecedentes familiares, a pesar de no tener una relación significativa, consideramos interesante que el 10,48 \% informó casos anteriores en la familia.

No hay duda de que los factores de riesgo cardiovascular están directamente asociados con este tipo de lesión y esto está claro en estudios que evaluaron la prevalencia de lesiones ateroscleróticas en grupos específicos, encontrando invariablemente una mayor incidencia de hallazgos como Ardakani et al. (2007) quienes encontraron calcificaciones en el $43 \%$ de los diabéticos o Ngmsom et al. y Friedlander et al. (2017), con calcificaciones en 38,9 
$\%$ y $31 \%$ de pacientes hipertensos, respectivamente. Quizás la hipertensión y el tabaquismo son los principales factores de riesgo para la formación de lesiones ateroscleróticas porque actúan, como se sabe, de manera negativa en la elasticidad de la pared del vaso. Johansson et al. afirmaron que los pacientes con calcificación carotídea tienen un riesgo $5,6 \%$ mayor de un resultado combinado de eventos vasculares.

Nuestro estudio tuvo una baja representatividad en dos factores de riesgo cardiovascular asociados, el tabaquismo y la hipertensión arterial. Con sólo el 12,5 \% de nuestros pacientes fumadores, no encontramos una relación significativa con la presencia de calcificaciones, número similar al de Ardakani et al. con 13,3\% y ligeramente por debajo de Atalay et al. con $27,11 \%$ y Johansson et al. y Lee et al. (2013) con $20 \%$.

Por otro lado, el 14,17 \% de nuestros casos eran hipertensos, estos datos mostraron una relación significativa con la presencia de calcificación en las carótidas, así como el estudio de Moshfeghi et al., quienes encontraron una relación significativa en el 21,2 \% de los casos. En contraste, los estudios con un mayor porcentaje de pacientes hipertensos como Johansson et al. con $82 \%$, Lee et al. (2013) con $42,3 \%$ y Ngamsom et al. con $36,52 \%$, no encontraron una relación significativa entre la hipertensión y la presencia de calcificaciones.

Nuestros resultados mostraron que el riesgo más alto se presenta en pacientes ancianos e hipertensos. Por lo tanto, enfatizamos la importancia de una observación exhaustiva del dentista en radiografías panorámicas en busca de cualquier alteración de la anatomía normal y, en el caso positivo, proceder a la referencia médica lo antes posible a un diagnóstico concluyente por parte de un especialista, pudiendo así ayudar en la prevención de episodios vasculares graves.

GARBELOTTI JUNIOR, S.; RIBEIRO MOREIRA, W.; AGUIAR, H.; OLAVE, E. \& QUEIROZ, D. Prevalence of atherosclerotic carotid lesions on panoramic radiography and relationship with cardiovascular risk factors in brazilians. Int. J. Morphol., 38(6):1560-1565, 2020.

SUMMARY: Although panoramic radiography is not an initial diagnostic test for detecting carotid atherosclerosis, we cannot ignore its value in viewing these lesions as incidental findings that can be great allies in early diagnosis and prevention of serious vascular events. The aim was to evaluate the prevalence of calcifications in the bifurcation of the carotid artery on routine panoramic radiographs as a diagnostic principle, as well as its relationship with cardiovascular risk factors in Brazilians. We analyzed 449 panoramic radiography exams from the database of the Department of Radiology of the Faculty of Dentistry of Piracicaba, UNICAMP, São Paulo, Brazil. After obtaining blood pressure measurements and information on smoking and systemic diseases, the researcher observed on the panoramic radiograph, the presence or absence of the radiopaque mass in the regions of the cervical vertebrae $\mathrm{C} 3-\mathrm{C} 4$. In the case of a suggestive image of atheroma, an anteroposterior radiography was performed to confirm the diagnosis. The Chi-square test of normal distribution for independent and random samples with a $95 \%$ confidence interval was applied to the data. Confirmation of carotid calcifications occurred in $8.5 \%$ of the study population (38 cases). We did not find any relationship between the presence of calcifications with smoking, sex or previous family history. However, $49.1 \%$ of cases occurred above sixty years old and there was a significant relationship with hypertension $(\mathrm{P}=0.006)$. The greatest risk was presented in elderly and hypertensive patients, so we highlight the importance of a thorough observation by the dentist of panoramic radiographs to detect any alteration of the normal anatomy and in the positive case, proceed with the referral as soon as possible for a conclusive diagnosis by a specialist, being able to thus assist in the prevention of severe vascular episodes.

KEY WORDS: Atherosclerosis; Carotid artery; Panoramic radiography; hypertension..

\section{REFERENCIAS BIBLIOGRÁFICAS}

Abreu, T. Q.; Ferreira, E. B.; Brito Filho, S. B.; Sales, K. P. F.; Lopes, F. F. \& Oliveira, A. E. F. Prevalence of carotid artery calcifications detected on panoramic radiographs and confirmed by Doppler ultrasonography: Their relationship with systemic conditions. Indian J. Dent. Res., 26(4):345-50, 2015.

Almog, D. M. Utility of panoramic radiographs in detecting cervical calcified carotid atheroma. Oral Surg. Oral Med. Oral Pathol. Oral Radiol. Endod., 104(4):451, 2007.

Almog, D. M.; Illig, K. A.; Carter, L. C.; Friedlander, A. H.; Brooks, S. L. \& Grimes, R. M. Diagnosis of non-dental conditions. Carotid artery calcifications on panoramic radiographs identify patients at risk for stroke. N. Y. State Dent. J., 70(8):20-5, 2004.

Almog, D. M.; Illig, K. A.; Elad, S.; Romano, P. R. \& Carter, L. C. Supplementary role of panoramic radiographs in the medical surveillance of a patient at risk for stroke. Compend. Contin. Educ. Dent., 26(6):369-72, 2005.

Ardakani, F. E.; Ardakani, M. A.; Mohammadi, Z. \& Sheikhha, M. H. Evaluating calcified carotid artery atheromas in panoramic radiographs of patients with type 2 diabetes mellitus. Oral Radiol., 23:6-9, 2007.

Atalay, Y.; Asutay, F.; Agacayak, K. S.; Koparal, M.; Adali, F. \& Gulsun, B. Evaluation of calcified carotid atheroma on panoramic radiographs and Doppler ultrasonography in an older population. Clin. Interv. Aging, 10:1121-9, 2015

Bastos, J. S.; Abreu, T. Q.; de Brito Filho, S. B.; de Sales, K. P. F.; Lopes, F. F. \& de Oliveira, A. E. F. Sensitivity and accuracy of panoramic radiography in identifying calcified carotid atheroma plaques. Braz. J. Oral Sci., 11(2):88-93, 2012.

Bayer, S.; Helfgen, E. H.; Bös, C.; Kraus, D.; Enkling, N. \& Mues, S. Prevalence of findings compatible with carotid artery calcifications on dental panoramic radiographs. Clin. Oral Investig., 15(4):563-9, 2011.

Bengtsson, V. W.; Persson, G. R. \& Renvert, S. Assessment of carotid calcifications on panoramic radiographs in relation to other used 
methods and relationship to periodontitis and stroke: a literature review. Acta Odontol. Scand., 72(6):401-12, 2014.

Bensenor, I. M.; Goulart, A. C.; Szwarcwald, C. L.; Vieira, M. L. F. P.; Malta, D. C. \& Lotufo, P. A. Prevalence of stroke and associated disability in Brazil: National Health Survey--2013. Arq. Neuropsiquiatr., 73(9):746-50, 2015.

Borba, D. L.; Hipólito, U. V. \& Pereira, Y. C. L. Early diagnosis of atherosclerosis with panoramic radiographs: a review. J. Vasc. Bras., 15(4):302-7, 2016.

Cohen, S. N.; Friedlander, A. H.; Jolly, D. A. \& Date, L. Carotid calcification on panoramic radiographs: an important marker for vascular risk. Oral Surg. Oral Med. Oral Pathol. Oral Radiol. Endod., 94(4):510-4, 2002.

Conselho Nacional de Secretários de Saúde (CONASS). Campanha Nacional de Combate ao AVC no Brasil em 2016. Brasilia, Conselho Nacional de Secretários de Saúde, 2016. Disponible en: http:// www.conass.org.br/campanha-nacional-de-combate-ao-avc-no-brasilem-2016/

Friedlander, A. H. \& Lande, A. Panoramic radiographic identification of carotid arterial plaques. Oral Surg. Oral Med. Oral Pathol., 52(1):1024, 1981.

Friedlander, A. H.; Aghazadehsanai, N.; Chang, T. I.; Harada, N. \& Garrett, N. R. Prevalence of calcified carotid artery atheromas on panoramic images of individuals with primary hyperparathyroidism. Dentomaxillofac. Radiol., 42(8):20130118, 2013.

Friedlander, A. H.; Garrett, N. R.; Chin, E. E. \& Baker, J. D. Ultrasonographic confirmation of carotid artery atheromas diagnosed via panoramic radiography. J. Am. Dent. Assoc., 136(5):635-40, 2005.

Friedlander, A. H.; Graves, L. L; Grabich, S. H; Aghazadehsanai, N. \& Chang, T. I. Prevalence of calcified carotid artery atheromas on panoramic images of older men with gout: a descriptive retrospective study. Dentomaxillofac Radiol., 46(5):20160406, 2017.

Garoff, M.; Ahlqvist, J.; Jäghagen, E. L.; Johansson, E. \& Wester, P. Carotid calcification in panoramic radiographs: radiographic appearance and the degree of carotid stenosis. Dentomaxillofac. Radiol., 45(6):20160147, 2016.

Gonçalves, J. R. S. N.; Yamada, J. L. Y.; Berrocal, C.; Westphalen, F. H.; Franco, A. \& Fernandes, A. Prevalence of pathologic findings in panoramic radiographs: calcified carotid artery atheroma. Acta Stomatol. Croat., 50(3):230-4, 2016.

Imanimoghaddam, M.; Rooh, M. R.; Hashemi, E. M \& Blouri, A. J. Doppler sonography confirmation in patients showing calcified carotid artery atheroma in panoramic radiography and evaluation of related risk factors. J. Dent. Res. Dent. Clin. Dent. Prospects, 6(1):6-11, 2012.

Johansson, E.; Ahlqvist, J.; Garoff, M.; Jäghagen, E. L.; Meimermondt, A. \& Wester, P. Carotid calcifications on panoramic radiographs: a 5-year follow-up study. Oral Surg. Oral Med. Oral Pathol. Oral Radiol., 120(4):513-20, 2015.

Lee, J. S.; Kim, O. S.; Chung, H. J.; Kim, Y. J.; Kweon, S. S.; Lee, Y. H.; Shin, M. H. \& Yoon, S. J. The correlation of carotid artery calcification on panoramic radiographs and determination of carotid artery atherosclerosis with ultrasonography. Oral Surg. Oral Med. Oral Pathol. Oral Radiol., 118(6):739-45, 2014.

Lee, J.S.; Kim, O. S.; Chung, H. J.; Kim, Y. J.; Kweon, S. S.; Lee, Y. H.; Shin, M. H. \& Yoon, S. J. The prevalence and correlation of carotid artery calcification on panoramic radiographs and peripheral arterial disease in a population from the Republic of Korea: the Dong-gu study. Dentomaxillofac. Radiol., 42(3):29725099, 2013.

Madden, R. P.; Hodges, J. S.; Salmen, C. W.; Rindal, D. B.; Tunio, J.; Michalowicz, B. S. \& Ahmad, M. Utility of panoramic radiographs in detecting cervical calcified carotid atheroma. Oral Surg. Oral Med. Oral Pathol. Oral Radiol. Endod., 103(4):543-8, 2007.

Ministério da Saúde do Brasil. AVC: O Que É, Causas, Sintomas, Tratamentos, Diagnóstico e Prevenção. Brasilia, Ministério da Saúde do Brasil, 2019. Disponible en: http://portalms.saude.gov.br/saude-dea-z/acidente-vascular-cerebral-avc

Moshfeghi, M.; Taheri, J. B.; Bahemmat, N.; Evazzadeh, M. E. \& Hadian,
H. Relationship between carotid artery calcification detected in dental panoramic images and hypertension and myocardial infarction. Iran. J. Radiol., 11(3):e8714, 2014.

Ngamsom, S.; Arayasantiparb, R.; Pornprasertsuk-Damrongsri, S. \& Sureephong, B. Relationship between calcified carotid atheromas in digital panoramic radiographs and underlying systemic diseases in implant patients. J. Investig. Clin. Dent., 6(4):301-6, 2015.

Ramesh, A. \& Pabla, T. Panoramic radiographs: a screening tool for calcified carotid atheromatous plaque. J. Mass. Dent. Soc., 56(2):20-1, 2007.

Ravon, N. A.; Hollender, L. G.; McDonald, V. \& Persson, G. R. Signs of carotid calcification from dental panoramic radiographs are in agreement with Doppler sonography results. J. Clin. Periodontol., 30(12):1084-90, 2003.

Roldán-Chicano, R.; Oñate-Sánchez, R. E.; López-Castaño, F.; CabrerizoMerino, M. C. \& Martínez-López, F. Panoramic radiograph as a method for detecting calcified atheroma plaques. Review of literature. Med. Oral Patol. Oral Cir. Bucal, 11(3):E261-6, 2006.

Yoon, S. J.; Shim, S. K.; Lee, J. S.; Kang, B. C.; Lim, H. J.; Kim, M. S. \& Kim, S. H. Interobserver agreement on the diagnosis of carotid artery calcifications on panoramic radiographs. Imaging Sci. Dent., 44(2):13741, 2014.

Yoon, S. J.; Yoon, W.; Kim, O. S.; Lee, J. S. \& Kang, B. C. Diagnostic accuracy of panoramic radiography in the detection of calcified carotid artery. Dentomaxillofac. Radiol., 37(2):104-8, 2008.

\author{
Dirección para correspondencia: \\ Silvio Antonio Garbelotti Junior \\ Departamento de Morfologia e Genética \\ Pós-graduação em Biologia Funcional e Estrutural \\ Universidade Federal de São Paulo \\ Rua Botucatu, 740 - Vila Clementino - 04023-900 \\ São Paulo - SP \\ BRASIL
}

Email: silviogarbelotti@gmail.com

Recibido : 10-04-2020

Aceptado: 15-06-2020 